Original Paper http://ajol.info/index.php/ijbcs http://indexmedicus.afro.who.int

\title{
Utilisation et vulnérabilité des espèces végétales et stratégies d'adaptation des populations riveraines de la Forêt Classée du Kou (Burkina Faso)
}

\author{
C. R. YAOVI*, M. HIEN, S. A. KABORE, Y. J. SEHOUBO et I. SOMDA \\ Institut du Développement Rural (IDR), Université Nazi Boni, 01 BP 1091 Bobo-Dioulasso, Burkina Faso. \\ *Auteur correspondant ; E-mail : yaoreca@gmail.com; Tel : +226 56081239.
}

Received: 28-11-2020 $\quad$ Accepted: 26-05-2021 $\quad$ Published: 30-06-2021

\section{RESUME}

La problématique de la déforestation est importante dans les régions arides et semi-arides de l'Afrique. Les politiques de gestion des forêts excluant les besoins réels des populations font qu'elle persiste. Cette étude vise à améliorer la gestion participative et durable des forêts classées au Burkina Faso. Une enquête ethnobotanique impliquant 60 ménages des villages riverains de la Forêt Classée du Kou et un inventaire floristique sur 36 placettes ont été réalisés. L'indice de vulnérabilité des espèces a été calculé et des analyses statistiques multidimensionnelles ont été effectuées. Les résultats montrent que la forêt comporte 206 espèces dont 80 utilisées pour l'alimentation (100\%), la médecine (96,7\%), l'artisanat (88,71\%), la construction $(88,7 \%)$, la bioénergie $(75,81 \%)$ et le fourrage $(64,52 \%)$. Les produits recherchés sont : fruits $(100 \%)$, feuilles $(95,16 \%)$ et bois $(93,55 \%)$. Le V-test et l'ACM révèlent une utilisation influencée par la profession, le sexe et le niveau académique. Des espèces utilisées, $68,75 \%$ sont vulnérables et $53,75 \%$ sont sur la liste rouge de l'UICN. Comme stratégies d'adaptation, $58,82 \%$ des enquêtés proposent l'agroforesterie et $41,18 \%$ proposent la substitution des espèces menacées. Il est indéniable que cette forêt classée couvre les besoins essentiels des populations riveraines qui doivent s'imposer une exploitation rationnelle de ses ressources.

(C) 2021 International Formulae Group. All rights reserved.

Mots clefs : Ethnobotanique, aires protégées, espèces vulnérables, conservation, Afrique de l'Ouest.

\section{Use and vulnerability of plant species and adaptation strategies of the riparian populations of the Kou Classified Forest (Burkina Faso)}

\begin{abstract}
The problem of deforestation is important in the arid and semi-arid regions of Africa. Forest management policies that exclude the real needs of the population justify the persistence of deforestation. This study aims to improve the participatory and sustainable management of classified forests in Burkina Faso. An ethnobotanical survey involving 60 households in the villages bordering the Kou Classified Forest and a floristic inventory on 36 plots were carried out. The species vulnerability index was calculated and multivariate statistical analyses were performed. The results show that the forest contains 206 species, 80 of which are used for food $(100 \%)$, medicine $(96.7 \%)$, handicrafts $(88.71 \%)$, construction $(88.7 \%)$, fuelwood $(75.81 \%)$ and fodder $(64.52 \%)$. The
\end{abstract}


products sought are: fruits (100\%), leaves $(95.16 \%)$ and wood $(93.55 \%)$. The V-test and the MCA reveal a use influenced by profession, gender and academic level. Of the species used, $68.75 \%$ are vulnerable and $53.75 \%$ are on the IUCN Red List. As adaptation strategies, $58.82 \%$ of the respondents propose agroforestry and $41.18 \%$ propose the substitution of threatened species by those available. It is undeniable that this classified forest covers the essential needs of the riparian populations who must impose themselves a rational exploitation of its resources.

(C) 2021 International Formulae Group. All rights reserved.

Keywords: Ethnobotany, protected areas, vulnerable species, conservation, West Africa.

\section{INTRODUCTION}

Dans les forêts tropicales et subtropicales, l'importance des produits forestiers non ligneux (PFNL) n'est plus à démontrer. En Afrique, plus des deux tiers de la population dépendent des ressources forestières pour leurs moyens de subsistance (CIFOR, 2005). Pour les populations rurales de l'Afrique sub-sahélienne, les PFNLs complètent la production agricole des ménages en leur apportant des denrées nutritionnelles essentielles, des produits à usage médicinal, du fourrage, de la paille, etc. (Loubelo, 2012). Ils sont pourvoyeurs des aliments de secours pendant la période de soudure. De ce fait, ils contribuent à accroitre la résilience de la population rurale face aux aléas saisonniers. Mais malheureusement cette dépendance des populations de ces ressources provoque de plus en plus la dégradation et la déforestation des écosystèmes forestiers pourvoyeurs de ces PFNL. En effet, pour nourrir cette population croissante, les surfaces agricoles cultivées augmentent partout au Sahel, les périodes de jachère diminuent et les rendements des cultures se dégradent (Ozer et al., 2007). Or, la diminution des revenus agricoles accentue la dépendance des populations aux emplois non agricoles tels que la collecte du bois de feu, des PFNLs et la production de charbon (Bauer, 2010). La question est encore plus cruciale autour des aires protégées, car la dégradation du couvert végétal au niveau des terroirs riverains aux aires protégées se solde par une pression accrue sur ces aires de conservation qui sont considérées comme des réservoirs importants de biodiversité (Hadonou-Yovo et al., 2019). Ainsi au Burkina Faso, 60\% du domaine des aires classées sont déjà occupés par des exploitations agricoles, des hameaux de cultures et même des villages dotés d'infrastructures socio-économiques (Tankoano et al., 2015). Situé dans la zone sahélo soudanienne, le Burkina Faso, ne dispose pas de vastes formations forestières. Seuls quelques îlots, en particulier les forêts classées et les parcs nationaux, subsistent encore et ne représentent que $14 \%$ du territoire national (DIFOR, 2007). C'est pour cela le gouvernement avec l'appui de ces partenaires a mis en place des politiques et des projets de gestion de ces aires protégées. Mais malheureusement, outre les principaux facteurs d'ordre climatique et anthropique connus, d'autres facteurs font que la déforestation persiste. Parmi ces facteurs, on peut citer les politiques de gestion des ressources forestières inadéquates ne prenant en compte ni les besoins réels des populations, ni le rôle social et économique des ressources forestières. A cause de ces lacunes, malgré leur coût, les projets et les plans de gestion forestiers, n'ont permis, ni d'inverser les tendances, ni de stopper le processus de dégradation des ressources forestières. Pour favoriser la prise en compte de ces informations dans les nouvelles politiques de gestion des ressources forestières, basées pour la plupart sur l'approche participative, plusieurs études ont été initiées (Hahn-Hadjali et Thiombiano, 2000; Lykke et al., 2004 ; Ouédraogo-Belem, 2008 ; Traoré et al., 2015 ; Savadogo, 2019). Elles restent insuffisantes de façon globale, voire quasi-inexistantes sur certaines aires protégées. C'est le cas au niveau de la Forêt Classée du Kou (FCK), considérée comme le poumon et le château d'eau de la ville de BoboDioulasso et de ses périphéries au Sud du Burkina Faso. Selon Guinko (2005), cette forêt est l'une des dernières reliques de forêt de type 
guinéen du pays et sa forêt galerie constitue une réserve écologique unique en son genre pour un pays sahélien. Vue la valeur écologique et socio-économique de cette forêt, il est important d'évaluer d'une part l'utilisation et la vulnérabilité des espèces végétales et d'autre part la perception et les stratégies d'adaptation de la population face à la perte de la diversité floristique. C'est pour cela cette étude a été initié au niveau FCK et vise à fournir des données pouvant améliorer et favoriser la gestion intégrée et participative des formations végétales subsistantes au sud du Burkina Faso.

\section{MATÉRIEL ET MÉTHODES \\ Zone d'étude}

L'étude a été réalisée dans la Forêt Classée du Kou (FCK) et dans deux villages riverains (Kokorowé et Nasso), situés à $15 \mathrm{~km}$ de la ville de Bobo-Dioulasso au Sud du Burkina Faso. La forêt s'étend entre $11^{\circ} 11^{\prime} 47^{\prime \prime}$ et $11^{\circ} 10^{\prime} 54^{\prime \prime}$ de latitude nord et entre $04^{\circ} 26^{\prime} 38^{\prime \prime}$ et $04^{\circ} 26^{\prime} 04^{\prime \prime}$ de longitude ouest (Figure 1). Elle couvre une superficie de 115 ha. Sa vocation principale est la protection de ses sources d'eau afin de ravitailler la ville de Bobo-Dioulasso en eau potable. De plus, on y note la récolte des produits forestiers non ligneux (PFNL) et l'écotourisme. Selon Thiombiano et al. (2010), le climat de la zone est de type Sud-Soudanien et est caractérisé par l'alternance d'une saison pluvieuse (mai à octobre) et d'une saison sèche (novembre à avril). La zone présente un relief essentiellement plat avec plusieurs unités pédologiques dont les principales sont : les sols hydromorphes, les sols ferrugineux tropicaux et les sols ferralitiques. La FCK appartient au domaine phytogéographique sud-soudanien et sa végétation est une mosaïque de formations forestières composées de forêt galerie, de forêt claire, des plantations, des savanes boisée, arborée et arbustive. Selon Ouédraogo et al. (2010), la présence et l'abondance de plusieurs espèces dans la FCK rappelle la composition floristique des forêts denses des zones guinéennes. Il s'agit de Anthocleista procera, Berlinia grandiflora, Calamus deerratus, Elaeis guineensis, Costus afer, et Anchomanes difformis . La forêt abrite également un site sacré où la population riveraine pratique des offices coutumiers. Cette population est composée majoritairement d'autochtones d'ethnie Bobo-vorè et quelques migrants d'ethnies Mossi, Peulh et autres. Ses principales activités sont: l'agriculture, l'élevage, l'exploitation des ressources forestières et fauniques, l'éco-tourisme, l'artisanat et le petit commerce. La commune de Bobo-Dioulasso où se trouve ces villages est la deuxième commune la plus peuplé du pays avec 983.552 habitants (INSD, 2020).

\section{Collecte des données}

Pour connaître les différentes utilisations faites des espèces végétales par la population riveraine de la FCK, la pression exercée sur les ressources forestières et les stratégies d'adaptation face à la perte de diversité floristique, une enquête ethnobotanique a été menée auprès de la population des villages de Nasso et de Kokorowé. L'enquête a été faite suivant un échantillonnage aléatoire stratifié (Houéhanou et al., 2016) avec un questionnaire direct à questions ouvertes et semi-structurées. Elle a été conduite auprès de 60 foyers dont 30 par village. Au total, 30 hommes et 30 femmes âgés d'au moins 20 ans ont été enquêtés. Le questionnaire a concerné l'usage des ressources végétales, les parties de la plante utilisée, les domaines d'utilisation, le mode de collecte, le stade de développement de la plante, le point de vue de l'informateur sur l'état d'abondance des espèces utilisées, leur perception des causes de la rareté des ressources végétales et les stratégies d'adaptation utilisées en cas de rareté ou de disparition des espèces végétales.

Pour évaluer le potentiel floristique et la disponibilité des espèces végétales dans la FCK, un inventaire forestier a été réalisé à l'intérieur de la forêt suivant un échantillonnage aléatoire stratifié avec un taux de sondage de $1,70 \%$. Ainsi pour l'inventaire des ligneux 24 placettes ont été installés soit 9 placettes de $500 \mathrm{~m}^{2}$ dans la forêt galerie et 7 placettes de $1000 \mathrm{~m}^{2}$ pour la savane arborée, 5 dans la savane arbustive, 2 dans la plantation de teck et 1 dans la forêt claire (Thiombiano et 
al., 2016). L'inventaire des herbacées a été fait au moment du maximum de phytomasse (novembre) sur 11 placettes à raison de 5 placettes dans la savane arbustive, 4 dans la savane arborée et 3 dans la forêt galerie. La méthode quantitative des points quadrats (Daget \& Poissonet, 1990) a été appliquée à l'aide d'un ruban de $20 \mathrm{~m}$ avec 100 point de contact, disposé su la longueur de la placette. La lecture a été répétée deux fois sur des lignes équidistantes de $10 \mathrm{~m}$, donnant ainsi 200 points de lecture. Dans chaque placette, un recensement exhaustif des espèces végétales a été effectué.

\section{Analyses des données}

La liste des espèces végétales inventoriées dans la FCK et celle des espèces utilisées par la population riveraine ont été établies. Les espèces végétales échantillonnées ont été identifiées à l'aide de la flore d'identification de Berhaut (1967) et Arbonnier (2009). Pour la composition floristique, la nomenclature de Lebrun et Stork (1991) a été utilisée. A partir des données recueillies les paramètres suivants ont été calculés :

- La Fréquence de Citation (FC) des utilisations et des organes utilisés a été exprimée suivant la formule :

$F C=\frac{n}{N} \times 100$

où $\mathbf{n}:$ Nombre d'informateurs ayant cité l'utilisation; $\mathbf{N}$ : nombre total d'informateurs.

- La Valeur d'Usage (VU) des espèces a été calculée suivant la formule simplifié de Cotton (1996) :

$V U=\sum_{i=1}^{i_{n}} \frac{U i}{N}$

$\mathbf{U}_{\mathbf{i}}$ : le nombre d'usages mentionnés par les informateurs, $\mathbf{N}$ : nombre total d'informateurs.

- L'Indice de Rareté (IR) a été calculé pour évaluer la disponibilité dans la FCK des espèces végétales utilisées par la population. La rareté reflète ici la fréquence à laquelle la plante a été observée dans la FCK. Il a été calculé selon l'équation suivante :
$I R=\left(1-\frac{n i}{N}\right) \times 100$

ni $=$ nombre de relevés dans lesquels l'espèce i est présente et $\mathbf{N}=$ nombre total de relevés. Les seuils d'interprétation utilisés par Traoré et al. (2011) sur les formations végétales soudaniennes ont été utilisés : IR< $60 \%$, pour les espèces très fréquentes dans les formations végétales ; $60 \leq \mathrm{IR}<80 \%$ pour les espèces moyennement fréquentes et IR $\geq 80 \%$ pour les espèces dites rares.

- L'Indice de Vulnérabilité de l'espèce $\boldsymbol{i}(\mathrm{IVi})$ a été calculé suivant la formule de Betti (2001) et est la moyenne des valeurs de 6 paramètres représentant des indicateurs majeurs de pression et de menace sur les espèces. La valeur de chaque paramètre $(\mathrm{P})$ varie de 1 à 3 suivant l'échelle de vulnérabilité de Betti (2001) modifié par Traoré et al. (2011) (Tableau 1). L'interprétation des IVi a été faite suivant les seuils d'interprétations proposés par Traoré et al. (2011) pour ligneux au Sud-Ouest du Burkina Faso : pour IVi $<2$ l'espèce est dite faiblement vulnérable; pour $2 \leq \mathrm{IVi}<\mathbf{2 , 5}$ l'espèce est moyennement vulnérable et pour IVi $\geq \mathbf{2 , 5}$ l'espèce est très vulnérable.

- Le statut au niveau national et international des espèces végétales utilisées par la population a été vérifié à partir de la liste rouge des espèces menacées de l'UICN (2020) et de celle des espèces menacées du Burkina Faso (Thiombiano et al., 2010).

- Des analyses statistiques multidimensionnelles ont été faites avec le logiciel Rstudio 3.3.6. Ainsi, une Analyse des Correspondances Multiples (ACM) et un Vtest de Cramer ont été appliqués aux données, afin d'identifier les facteurs socioprofessionnelles (village, sexe, niveau d'études, profession) qui influencent l'utilisation des espèces végétales. Une Analyse en Composantes Principales (ACP) et une matrice de corrélation ont été faites afin d'appréhender les relations entre les catégories d'usage, les produits de la plante et la dynamique des espèces utilisées. 

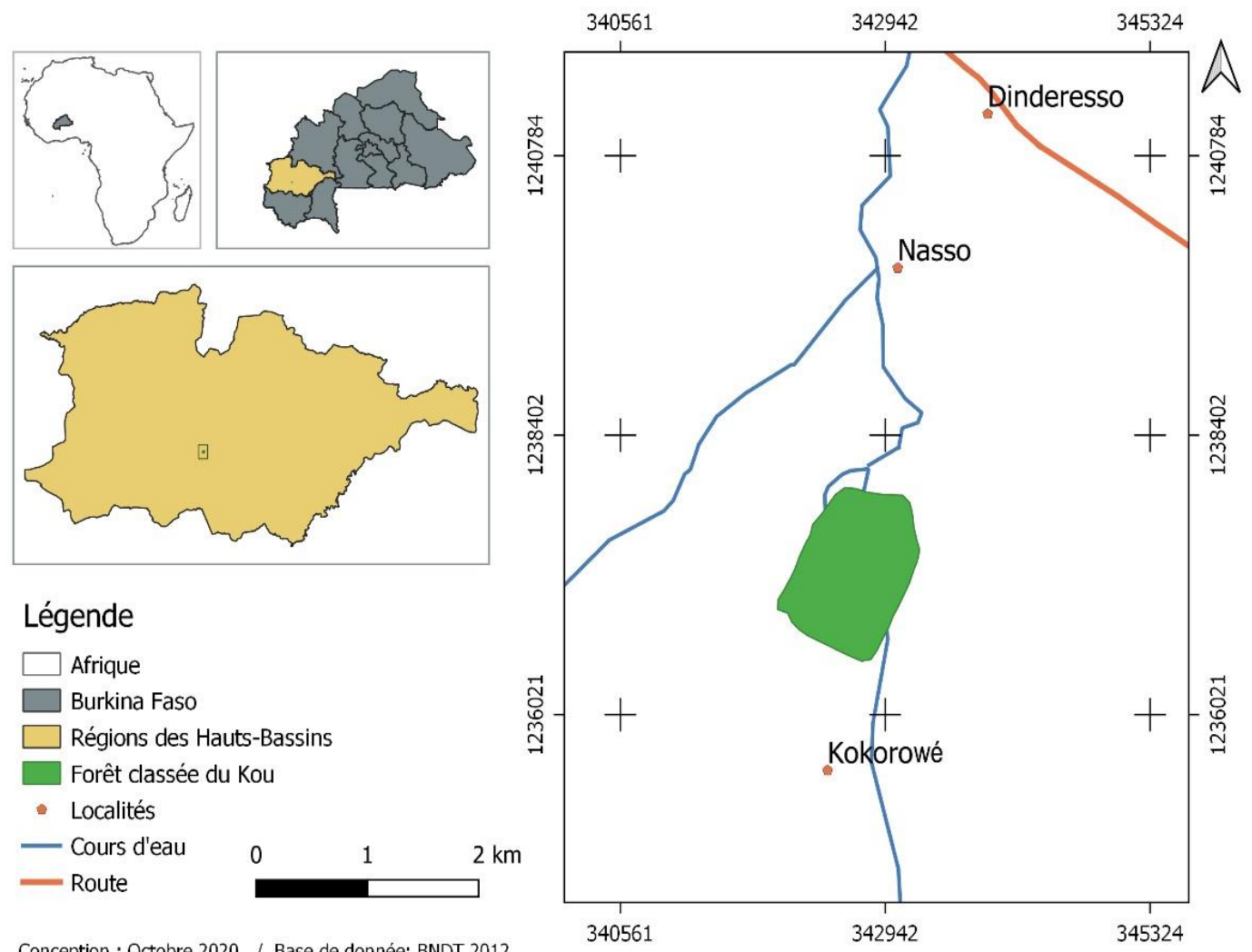

Conception : Octobre 2020 / Base de donnée: BNDT 2012

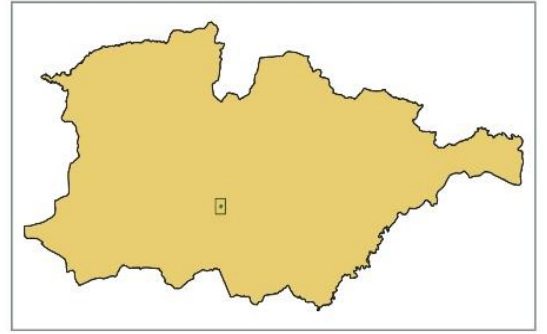

Légende

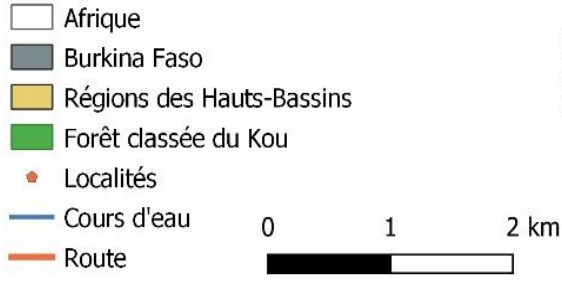

Figure 1 : Carte de localisation de la forêt classée du Kou et des villages de Nasso et de Kokorowé.

Tableau 1 : Paramètres importants pris en compte pour le calcul d'indice de vulnérabilité.

\section{Vulnérabilité a une exploitation incontrôlée}

\section{Paramètres}

\section{Faible (échelle=1) Moyenne (échelle=2) Forte (échelle=3)}

\begin{tabular}{lccc}
\hline P1 : Fréquence d'utilisation & $\mathrm{Fu}<20 \%$ & $20 \% \leq \mathrm{Fu} \leq 60 \%$ & $\mathrm{Fu} \geq 60 \%$ \\
\hline P2 : Nombre d'usage & $\mathrm{Nu}<2$ & $2 \leq \mathrm{Nu} \leq 4$ & $\mathrm{Nu} \geq 5$ \\
\hline P3 : Organe végétal utilisé & Feuille, latex & Fruits, branches & $\begin{array}{c}\text { Racine, graine, } \\
\text { écorce, fleur, bois }\end{array}$ \\
\hline P4 : Mode de collecte & Ramassage & - & Cueillette, coupe \\
\hline P5 : Stade de développement & Vieux ou sénescent & Adulte & Jeune \\
\hline P6 : Fréquence relative & $\mathrm{Fr} \geq 2 / 3 \mathrm{Fm}$ & $1 / 3 \mathrm{Fm} \leq \mathrm{Fr}<2 / 3 \mathrm{Fm}$ & $\mathrm{Fr}<1 / 3 \mathrm{Fm}$ \\
\hline
\end{tabular}

$\mathbf{F u}$ : Fréquence d'utilisation relative (nombre de personnes ayant cité l'espèce $i$ sur le nombre total d'informateurs); $\mathbf{N u ~ : ~}$

Nombre d'usages, Fr : Fréquence relative (nombre de relevés où l'espèce est présente sur nombre total de relevés); Fm :

Fréquence relative maximale. 


\section{RESULTATS}

\section{Diversité des espèces utilisées et disponibles dans la FCK}

Au total 80 espèces végétales réparties en 72 genres et en 32 familles ont été recensées lors de l'enquête ethnobotanique (Tableau 2). Lors de l'inventaire, 206 espèces réparties en 62 familles et 164 genres ont été recensées dans la FCK. Par rapport à la composition floristique, on note au niveau de la flore utilisée, une prédominance des Fabaceae $(23,46 \%)$ suivis des Malvaceae $(7,41 \%)$ et Combretaceae $(7,41 \%)$ (Figure 2). Dans la flore de FCK ce sont également les Fabaceae $(18,45 \%)$ qui dominent suivis des Rubiaceae $(7,77 \%)$, des Poaceae $(7,28 \%)$ et des Malvaceae $(6,80 \%)$. Quant à la disponibilité, $74,04 \%$ des espèces utilisées par les populations riveraines sont présentes dans la flore de la FCK. Parmi celles-ci, l'indice de rareté montre que $57,50 \%$ sont rares dans la FCK (IR $\geq 80 \%$ ) contre seulement $10 \%$ fréquentes et $5 \%$ sont très fréquentes.

\section{Utilisation des espèces végétales par la population riveraine de la FCK}

Les 80 espèces recensées sont utilisées dans 6 catégories d'usages et les fréquences de citation se présentent comme suit : l'alimentation (100\%), la médecine $(96,7 \%)$, l'artisanat $(88,71 \%)$, la construction $(88,7 \%)$, la bioénergie $(75,81 \%)$ et le fourrage $(64,52 \%)$. Les produits ou organes les plus sollicités sont les fruits ( $\mathrm{Fc}=100 \%$ des enquêtés), les feuilles $(95,16 \%)$, et les bois $(93,55 \%)$. Les autres produits sont les racines $(53,23 \%)$, les graines $(48,39 \%)$, les écorces $(43,55 \%)$ et la plante entière $(25,81 \%)$. Les valeurs d'usage (VU) des espèces utilisées varient entre 0,22 et 2,27. Les espèces les plus prisées $(\mathrm{VU} \geq 1)$ par les populations sont : Vitellaria paradoxa $(2,27)$, Borassus akeassii (1,57), Parkia biglobosa $(1,45)$ et Detarium microcarpum $(1,23)$.

L'analyse en composantes principales (ACP) concernant les catégories d'usage et les produits utilisés (Figure 3) montre que les deux premiers axes contribuent à $84,47 \%$ des informations analysées (Axe $1=69,41 \%$ et $\mathrm{Axe}_{2}=15,00 \%$ ). Il ressort de l'interprétation de l'axe $_{1}$ que les fruits sont essentiellement utilisés dans l'alimentation; les écorces, les racines et les graines en médecine; les bois pour la construction et l'artisanat et enfin la plante entière pour le fourrage.

La matrice des corrélations (Figure 4) montre qu'en plus des racines et des écorces, la plante entière est utilisée en médecine. Les bois sont plus utilisés comme bioénergie et les feuilles sont plus utilisées en médecine et en artisanat qu'en alimentation. La matrice met également en évidence le lien entre l'utilisation des espèces et la perception des populations sur l'importance, la disponibilité et la dynamique des espèces végétales utilisées. Elle révèle que la dynamique des espèces, perçue par la population, est négativement corrélée aux catégories d'usage et à toutes les formes d'exploitation des plantes et plus particulièrement à l'utilisation médicinale et à l'exploitation des feuilles, des racines et des écorces.

\section{Influence des facteurs socio-professionnels sur l'utilisation des espèces végétales}

Les enquêtés dans les villages de Nasso et de Kokorowé sont d'ethnie «bobo » et leur âge varie entre 20 et 83 ans. Les catégories socio-professionnelles identifiées sont : cultivateur/trice $(66,13 \%)$, «dolotière »: productrice de bière locale $(17,74 \%)$, ménagère (6,45\%), employé (4,84\%), guide écotouristique $(3,23 \%)$, transformatrice de produits agricoles $(1,61 \%)$ et phytothérapeute $(1,61 \%)$. Parmi eux $50 \%$ ne sont pas instruits, $40,32 \%$ ont fait le primaire et $9,68 \%$ le secondaire.

L'Analyse des Correspondances Multiples (ACM) réalisée met en évidence les liens entre l'utilisation des espèces et les facteurs socio-professionnels (Figure 5). Elle montre que l'utilisation de beaucoup d'espèces végétales (bcps_sp) est liée à la profession "dolotière (productrice de bière locale)", au sexe "féminin" et au niveau académique "non instruit". Les deux premiers axes de l'ACM contribuent à $30,1 \%$ des informations analysées.

Le V-test de Cramer quantifie le lien entre les modalités de la variable à expliquer (utilisation des espèces) et les modalités des 
autres variables explicatrices (facteurs socioprofessionnelles). Les résultats montrent que l'utilisation de beaucoup d'espèces végétales (bcps_sp) a un fort lien (V-test $\geq 1$ ) avec la profession "dolotière: productrice de bière locale" (V-test $=2,55 ; \quad p=0,01)$, le sexe "féminin" (V-test $=1,90 ; p=0,06)$ et le niveau académique "non-instruit" (V-test $=1,12$; $\mathrm{p}=0,26)$. L'utilisation de peu d'espèces (peu_sp) a un fort lien positif avec le sexe "homme" (V-test $=1,54 ; p=0,12)$ et l'âge "plus de 60 ans" (V-test=1,24; $\mathrm{p}=0,21)$. Mais seul le V-test de la profession «dolotière » est significatif $(\mathrm{p}<0,05)$.

\section{Vulnérabilité relative des espèces végétales utilisées}

Les indices de vulnérabilité (IVi) calculés ont permis de réaliser le spectre de vulnérabilité des espèces utilisées (Figure 6). Les résultats montrent que $68,75 \%$ des espèces utilisées (soit 55 espèces) possèdent un indice de vulnérabilité supérieur ou égal à 2 et sont ainsi dites vulnérables. Parmi ces espèces, $17,5 \%$ (14 espèces) sont très vulnérables (IV $\geq$ 2,5). Les $31,25 \%$ restantes (25 espèces) sont faiblement vulnérables. Par rapport aux facteurs de vulnérabilité, $83 \%$ des espèces utilisées sont vulnérables à cause du mode de collecte (coupe et/ou cueillette) et $76 \%$ à cause de la nature des organes prélevés (le bois, les graines, l'écorces, les racines et/ou les fleurs). Par contre la vulnérabilité de certaines espèces comme Parkia biglobosa et Detarium microcarpum est dû aussi au fait qu'elles sont utilisées dans toutes les catégories d'usages identifiés.

\section{Statut des espèces végétales utilisées par les populations}

La vérification des statuts des espèces végétales utilisées par la population riveraine de la FCK montre que $37,50 \%$ des espèces sont sur la liste des espèces menacées du Burkina Faso et 53,75\% (43 espèces) sur la liste rouge de l'UICN avec 38 espèces à préoccupation mineure (LC), 1 espèce quasi menacée (Eucalyptus camaldulensis), 3 espèces vulnérables (Khaya senegalensis, Vitellaria paradoxa et Afzelia africana) et 1 espèce en danger (Pterocarpus erinaceus).

\section{Perception de la perte de la biodiversité et stratégies d'adaptation des populations}

Les populations riveraines de la FCK sont conscientes de la vulnérabilité des ressources forestières dans leur localité. En effet, $71,43 \%$ des informateurs reconnaissent que les espèces végétales sont vraiment menacées de disparition. Quant à la perception des causes, 50,70\% des informateurs pensent qu'elles sont strictement anthropiques, 35,21\% pensent qu'elles sont à la fois climatiques et anthropiques et seuls $14,08 \%$ pensent qu'elles sont strictement climatiques.

Face à la raréfaction ou la disparition des espèces végétales, 58,82\% des informateurs proposent comme stratégie d'adaptation l'agroforesterie et $41,18 \%$ proposent la substitution des espèces menacées par d'autres espèces disponibles. Au total 11 espèces de substitution ont été recensées lors de l'enquête. Ce sont: Anacardium occidentale, Tectona grandis, Senna siamea, Eucalyptus camaldulensis, Azadirachta indica, Terminalia avicennioides, Vitellaria paradoxa, Glycine maxima, Gmelina arborea, Parinari curatellifolia et Parkia biglobosa.

Tableau 2 : Liste des espèces végétales utilisées par les populations riveraines de la FCK.

\begin{tabular}{lcllllll}
\hline \multicolumn{1}{c}{ Espèces } & $\begin{array}{c}\text { FC } \\
\text { K }\end{array}$ & Utilisations & $\begin{array}{c}\text { Partie ou } \\
\text { organes utilisés }\end{array}$ & VU & IVi & IR & Statut \\
\hline Acacia dudgeoni & + & $\mathrm{Me}$ & $\mathrm{Gr}$ & 0,03 & 1,83 & 95,83 & \\
\hline Acacia senegalensis & + & $\mathrm{Me}$ & $\mathrm{Ra}$ & 0,02 & 2,00 & 95,83 & \\
\hline Adansonia digitata & - & $\mathrm{Al} ; \mathrm{Me} ; \mathrm{Ar} ;$ & $\mathrm{Fe} ; \mathrm{Fr} ; \mathrm{Bo} ; \mathrm{Ra} ; \mathrm{Ec}$ & 0,48 & 2,67 & - & $M$ \\
\hline
\end{tabular}




\begin{tabular}{|c|c|c|c|c|c|c|c|c|}
\hline Afzelia africana & + & Me; Fo; Ar; Bé & $\mathrm{Fe} ; \mathrm{Bo} ; \mathrm{Ra} ; \mathrm{Gr}$ & 0,08 & 2,33 & 83,33 & $M$ & $V U$ \\
\hline Agelanthus dodoneifolius & + & $\mathrm{Al} ; \mathrm{Me} ; \mathrm{Ar} ;$ & Fr; Bo; Ec; Gr & 0,15 & 2,17 & 95,83 & & \\
\hline Anacardium occidentale & - & $\mathrm{Al} ; \mathrm{Me}$ & $\mathrm{Fe} ; \mathrm{Fr} ; \mathrm{Gr}$ & 0,13 & 2,00 & - & & \\
\hline Andropogon gayanus & + & Fo; $\mathrm{Ar}$ & $\mathrm{Fe}$ & 0,03 & 1,83 & 95,83 & & \\
\hline Annona senegalensis & + & Al; Me; Fo; Bé & $\begin{array}{l}\mathrm{Fe} ; \mathrm{Fr} ; \mathrm{Bo} ; \mathrm{Ra} ; \\
\mathrm{Ec} ; \mathrm{Gr}\end{array}$ & 0,61 & 2,67 & 95,83 & & $L C$ \\
\hline Anogeissus leiocarpus & + & Me; Fo; Ar; Bé; Co & $\begin{array}{l}\text { Fe; Bo; Ra; Ec; } \\
\mathrm{Gr}\end{array}$ & 0,56 & 2,33 & 37,50 & $M$ & $L C$ \\
\hline Arachis hypogaea & - & Fo & Fe; Bo & 0,02 & 1,83 & - & & \\
\hline Azadirachta indica & + & Me; Ar; Bé; Co & $\mathrm{Fe} ; \mathrm{Bo} ; \mathrm{Ec} ; \mathrm{Gr}$ & 0,18 & 2,33 & 95,83 & & $L C$ \\
\hline Balanites aegyptiaca & - & $\mathrm{Al} ; \mathrm{Me}$ & Fr; Gr & 0,03 & 1,67 & - & & $L C$ \\
\hline Berlinia grandiflora & + & Al; Me; Bé; Co & $\mathrm{Fe} ; \mathrm{Bo} ; \mathrm{Ra} ; \mathrm{Gr}$ & 0,08 & 2,33 & 91,67 & & $L C$ \\
\hline Blighia sapida & - & $\mathrm{Al}$ & Fr & 0,02 & 1,67 & - & & \\
\hline Bombax costatum & + & Al; Ar; Bé; Co & Fe; Fr; Bo; Gr & 0,32 & 2,67 & 95,83 & $M$ & $L C$ \\
\hline Borassus akeassii & + & Al; Me; Ar; Bé; Co & $\mathrm{Fe} ; \mathrm{Fr} ; \mathrm{Bo} ; \mathrm{Ra} ; \mathrm{Gr}$ & 1,52 & 2,83 & 91,67 & $M$ & $L C$ \\
\hline Burkea africana & + & Me; Ar; Bé; Co & Bo; Ra; Ec & 0,19 & 2,00 & 95,83 & & $L C$ \\
\hline Carapa procera & + & Al; Me; Bé; & Fe; Fr; Bo; Ec & 0,06 & 2,17 & 75,00 & & $L C$ \\
\hline Cassia sieberiana & - & $\mathrm{Me}$ & $\mathrm{Ra}$ & 0,02 & 2,17 & - & & $L C$ \\
\hline Senna siamea & + & Ar, Bé, Mé & Bo, Ra & 0,02 & 1,83 & 95,83 & & \\
\hline Ceiba pentandra & + & $\mathrm{Al} ; \mathrm{Me} ; \mathrm{Ar} ;$; Co & Fe; Fr; Bo; Gr & 0,48 & 2,50 & 91,67 & $M$ & $L C$ \\
\hline Celtis integrifolia & + & Fo; Bé & Fe; Bo & 0,03 & 2,33 & 91,67 & $M$ & \\
\hline Cochlospermum planchonii & & $\mathrm{Me}$ & Bo & 0,02 & 2,00 & 95,83 & & \\
\hline Cola cordifolia & + & $\mathrm{Al} ; \mathrm{Ar}$; & Fr; Bo & 0,03 & 2,33 & 91,67 & & $L C$ \\
\hline Combretum adenogonium & + & Me; Bé & Fe; Bo & 0,06 & 2,17 & 79,17 & $M$ & $L C$ \\
\hline Cordia myxa & + & Al; Me; Fo, Ar; Bé; Co & $\begin{array}{l}\mathrm{Fe} ; \mathrm{Fr} ; \mathrm{Bo} ; \mathrm{Ra} ; \\
\mathrm{Ec} ; \mathrm{Gr}\end{array}$ & 0,73 & 2,83 & 95,83 & & \\
\hline Crossopteryx febrifuga & + & Me; Bé; Co & $\mathrm{Fe} ; \mathrm{Bo} ; \mathrm{Ra} ; \mathrm{Gr}$ & 0,16 & 2,33 & 91,67 & $M$ & $L C$ \\
\hline Daniellia oliveri & & Me; Fo; Ar; Bé & Fe; Fr; Bo & 0,19 & 2,33 & 87,50 & $M$ & $L C$ \\
\hline Detarium microcarpum & + & $\mathrm{Al} ; \mathrm{Me} ; \mathrm{Bé} ; \mathrm{Co}$ & $\begin{array}{l}\text { Fe; Fr; Bo; Ra; } \\
\text { Ec; Gr }\end{array}$ & 1,19 & 2,67 & 87,50 & $M$ & $L C$ \\
\hline Dichrostachys cinerea & & $\mathrm{Me}$ & $\mathrm{Gr}$ & 0,02 & 1,83 & 95,83 & & $L C$ \\
\hline Diospyros mespiliformis & + & $\mathrm{Al} ; \mathrm{Me} ; \mathrm{Fo} ; \mathrm{Ar} ; \mathrm{Co}$ & $\begin{array}{l}\mathrm{Fe} ; \mathrm{Fr} ; \mathrm{Bo} ; \mathrm{Ra} ; \\
\mathrm{Ec} ; \mathrm{Gr}\end{array}$ & 0,53 & 2,67 & 70,83 & & \\
\hline Elaeis guineensis & + & $\mathrm{Al} ; \mathrm{Ar}$; & $\mathrm{Fe} ; \mathrm{Gr}$ & 0,18 & 1,83 & 54,17 & $M$ & $L C$ \\
\hline Entada africana & & Me; Ar; Bé; Co & Fe; Bo & 0,08 & 2,17 & 79,17 & & $L C$ \\
\hline Eucalyptus camaldulensis & - & Me; Ar; Bé; Co & Fe; Bo & 0,24 & 2,17 & - & & $N T$ \\
\hline Faidherbia albida & & Me, Fo; Ar; Bé & Fe; Fr; Bo; Ra; Ec & 0,13 & 2,17 & - & & $L C$ \\
\hline Ficus sp. & + & Me; Fo & Fr; Bo; Gr & 0,06 & 2,33 & 95,83 & & \\
\hline Ficus sycomorus & & $\mathrm{Al} ; \mathrm{Me} ; \mathrm{Fo}$ & $\mathrm{Fe} ; \mathrm{Fr}$ & 0,18 & 2,17 & 95,83 & & $L C$ \\
\hline Gardenia erubescens & + & $\mathrm{Al}$ & Fr & 0,05 & 1,50 & 95,83 & $M$ & \\
\hline Gardenia ternifolia & & $\mathrm{Me}$ & $\mathrm{Fe} ; \mathrm{Fr}$ & 0,16 & 2,00 & 91,67 & & $L C$ \\
\hline Gmelina arborea Roxb. & & Fo; Ar; Bé; Co & Fe; Bo & 0,24 & 2,33 & 91,67 & & $L C$ \\
\hline Grewia bicolor & - & $\mathrm{Al}$ & Ec & 0,02 & 2,17 & - & & \\
\hline Guiera senegalensis & & $\mathrm{Me}$ & $\mathrm{Fe} ; \mathrm{Ra}$ & 0,03 & 2,17 & 95,83 & & $L C$ \\
\hline Hymenocardia acida & + & Al; Me; Bé; Co & Fe; Fr; Bo; Ra & 0,18 & 2,17 & 95,83 & & $L C$ \\
\hline Khaya senegalensis & + & Me; Fo; Ar; Bé; Co & Fe; Bo; Ra; Ec & 0,79 & 2,67 & 37,50 & $M$ & $V U$ \\
\hline
\end{tabular}




\begin{tabular}{|c|c|c|c|c|c|c|c|c|}
\hline Landolphia heudelotii & - & $\mathrm{Al} ; \mathrm{Me} ; \mathrm{Ar}$ & $\mathrm{Fe} ; \mathrm{Fr} ; \mathrm{Bo} ; \mathrm{Gr}$ & 0,15 & 2,33 & - & $M$ & \\
\hline Lannea microcarpa & - & Al; Me; Fo; Bé; Co & Fe; Fr; Bo; Ra; Ec & 0,40 & 2,67 & - & $M$ & $L C$ \\
\hline Lannea velutina & + & $\mathrm{Al}$ & Fr & 0,03 & 1,67 & 91,67 & & $L C$ \\
\hline Mangifera indica & - & $\mathrm{Al} ; \mathrm{Me}$ & Fr; Ec & 0,05 & 1,83 & - & & \\
\hline Moringa oleifera & - & Al; Me; Bé; & $\mathrm{Fe}$ & 0,23 & 2,17 & - & & \\
\hline Musa paradisiaca & - & $\mathrm{Al} ; \mathrm{Me} ;$ & Fr; Ra & 0,03 & 2,00 & - & & \\
\hline Ocimum basilicum & - & $\mathrm{Me}$ & $\mathrm{Fe}$ & 0,02 & 1,17 & - & & \\
\hline Ocimum canum & - & $\mathrm{Me}$ & $\mathrm{Fe}$ & 0,02 & 1,33 & - & & \\
\hline Opilia celtidifolia & - & Fo & $\mathrm{Fe}$ & 0,02 & 1,17 & - & & \\
\hline Oxytenanthera abyssinica & + & Me; Ar; & Fe; Bo & 0,06 & 2,17 & 95,83 & & \\
\hline Parinari curatellifolia & + & Me; Bé & Bo; Ec & 0,06 & 2,00 & 95,83 & $M$ & $L C$ \\
\hline Parkia biglobosa & + & Al; Me; Fo, Ar; Bé; Co & $\begin{array}{l}\text { Fe; Fr; Bo; Ra; } \\
\text { Ec; Gr }\end{array}$ & 1,40 & 2,83 & 91,67 & $M$ & $L C$ \\
\hline Paullinia pinnata & + & $\mathrm{Al} ; \mathrm{Ar} ; \mathrm{Co}$ & Bo & 0,05 & 2,33 & 95,83 & & \\
\hline Piliostigma thonningii & + & Me; Fo; & Fe; Fr; Ra; Gr & 0,10 & 2,17 & 79,17 & & \\
\hline Potamogeton octendrus & + & Fo & $\mathrm{Fe}$ & 0,03 & 1,50 & 95,83 & & \\
\hline Prosopis africana & + & Fo; Ar; Bé; Co & Fe; Bo & 0,13 & 2,33 & 87,50 & $M$ & $L C$ \\
\hline Pteleopsis suberosa & + & Al; Me; Bé & $\mathrm{Fe} ; \mathrm{Bo} ; \mathrm{Ra} ; \mathrm{Ec}$ & 0,24 & 2,50 & 95,83 & $M$ & $L C$ \\
\hline Pterocarpus erinaceus & + & Al; Fo; Ar; Bé; Co & Fe, Fr; Bo & 0,53 & 2,67 & 62,50 & $M$ & $E N$ \\
\hline saba comorensis & + & $\mathrm{Al} ; \mathrm{Me}$ & $\mathrm{Fe} ; \mathrm{Fr}$ & 0,06 & 1,83 & 87,50 & & \\
\hline Saba senegalensis & + & $\mathrm{Al} ; \mathrm{Me} ; \mathrm{Ar}$ & Fe; Fr; Ra & 0,34 & 2,00 & 58,33 & $M$ & \\
\hline Sarcocephalus latifolius & & $\mathrm{Me}$ & $\mathrm{Fe}$ & 0,02 & 1,33 & 91,67 & & \\
\hline Sclerocarya birrea & + & $\mathrm{Al} ; \mathrm{Me}$ & $\mathrm{Fe} ; \mathrm{Fr}$ & 0,11 & 1,83 & 95,83 & $M$ & \\
\hline $\begin{array}{l}\text { Securidaca } \\
\text { longipedunculata }\end{array}$ & + & $\mathrm{Al} ; \mathrm{Me} ; \mathrm{Co}$ & Fe, Fr; Bo; Ra & 0,11 & 2,33 & 95,83 & $M$ & \\
\hline Senna alata & + & Me; & $\mathrm{Fe}$ & 0,02 & 1,67 & 95,83 & & $L C$ \\
\hline Senna occidentalis & + & $\mathrm{Me}$ & $\mathrm{Fe}$ & 0,02 & 1,33 & 95,83 & & \\
\hline Sterculia setigera & + & Me; & $\mathrm{Fe}$ & 0,03 & 1,67 & 91,67 & $M$ & \\
\hline Strophanthus sarmentosus & & Me; & $\mathrm{Fe}$ & 0,02 & 1,67 & 87,50 & & \\
\hline Strychnos spinosa & - & $\mathrm{Al}$ & $\mathrm{Fe} ; \mathrm{Fr}$ & 0,10 & 1,50 & - & & \\
\hline Tamarindus indica & - & Al; Me; Ar; Bé; Co & Fe, Fr; Bo & 0,19 & 2,17 & - & $M$ & $L C$ \\
\hline Tectona grandis & + & Me; Ar; Bé; Co & $\mathrm{Fe} ; \mathrm{Bo}$ & 0,45 & 2,33 & 70,83 & & \\
\hline Terminalia avicennioides & + & Al; Me; Ar; Bé; Co & $\mathrm{Fe}, \mathrm{Fr} ; \mathrm{Bo} ; \mathrm{Ra}$ & 0,56 & 2,67 & 95,83 & & $L C$ \\
\hline Trichilia emetica & & $\mathrm{Me}$ & $\begin{array}{l}\mathrm{Fe} ; \mathrm{Fr} ; \mathrm{Bo} ; \mathrm{Ra} ; \\
\mathrm{Ec} ; \mathrm{Gr}\end{array}$ & 0,03 & 1,83 & 95,83 & & $L C$ \\
\hline Vitellaria paradoxa & + & $\mathrm{Al} ; \mathrm{Me} ; \mathrm{Ar} ; \mathrm{Bé} ; \mathrm{Co}$ & Fe, Fr; Bo; Ra & 2,19 & 2,67 & 75,00 & $M$ & $V U$ \\
\hline Vitex doniana & + & Al; Me; Fo, Ar; Bé; Co & Fe; Fr; Ra; Gr & 0,42 & 2,67 & 83,33 & $M$ & $L C$ \\
\hline Ximenia americana & + & $\mathrm{Al} ; \mathrm{Me} ; \mathrm{Fo}$ & $\mathrm{Fe} ; \mathrm{Fr}$ & 0,19 & 1,83 & 95,83 & $M$ & $L C$ \\
\hline Ziziphus mauritiana & - & & $\mathrm{Fe}$ & 0,05 & 2,00 & - & $M$ & $L C$ \\
\hline
\end{tabular}

FCK : Forêt Classée du Kou ; + : espèces présente dans la FCK ; - : espèces absente dans la FCK

Utilisation (Al : Alimentaire; Me : Médecine ; Fo : Fourrage, Ar : Artisanat ; Bé : Bioénergie ; C : Construction) ; Organe ou partie utilisé (Fe : Feuille, Fr : Fruit, Bo : bois, Ti : Tige, R : Racine, Ec : écorce, Gr : Graine) ; Statut (M : Menacée de disparition au Burkina Faso ; LC : espèce à préoccupation mineur, $\mathbf{V U}$ : espèce vulnérable, $\mathbf{N T}$ : espèces quasi menacée et EN : espèce en danger sur la liste rouge de UICN). 


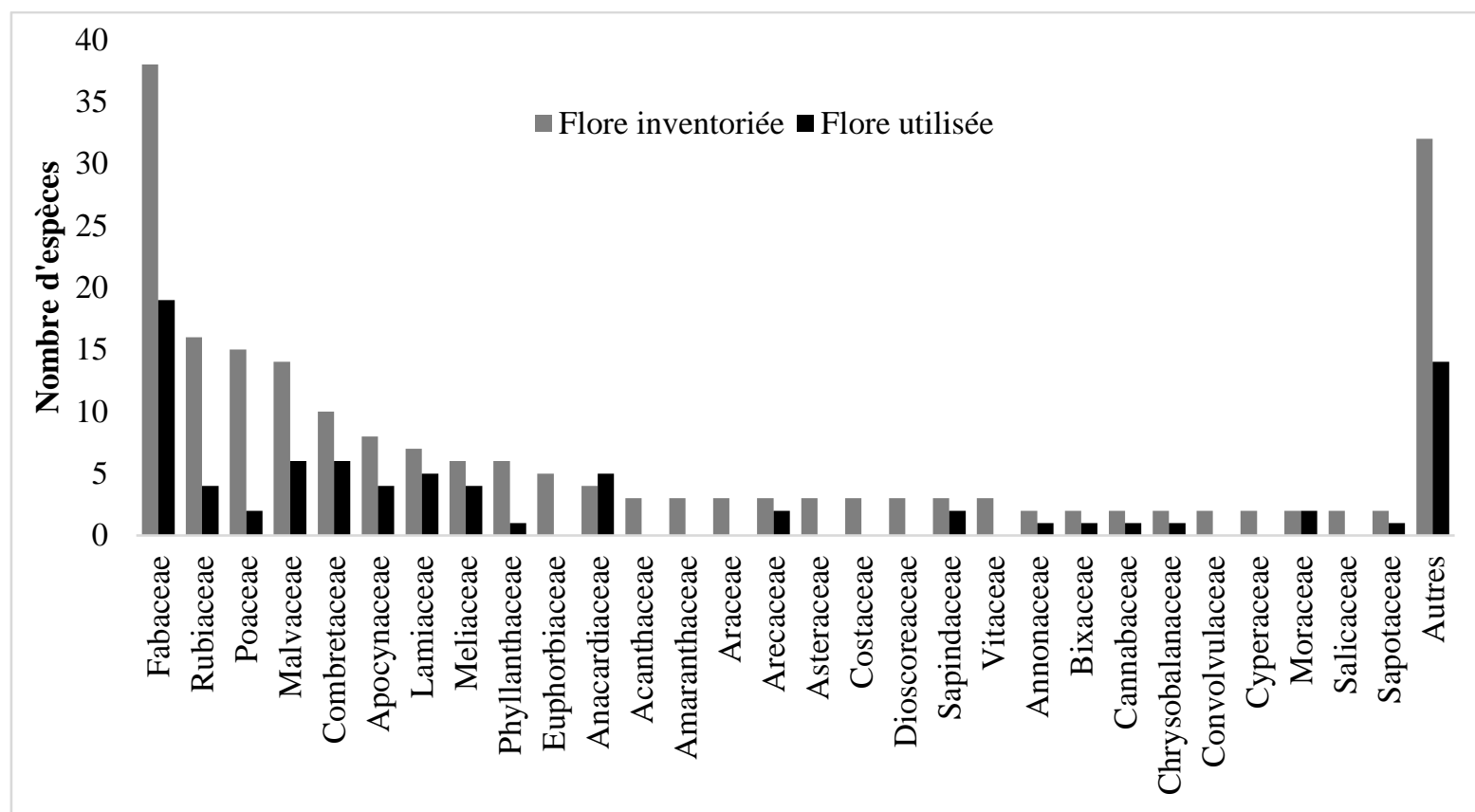

Familles

Figure 2 : Spectre comparé des familles utilisées et celles inventoriées dans la FCK.

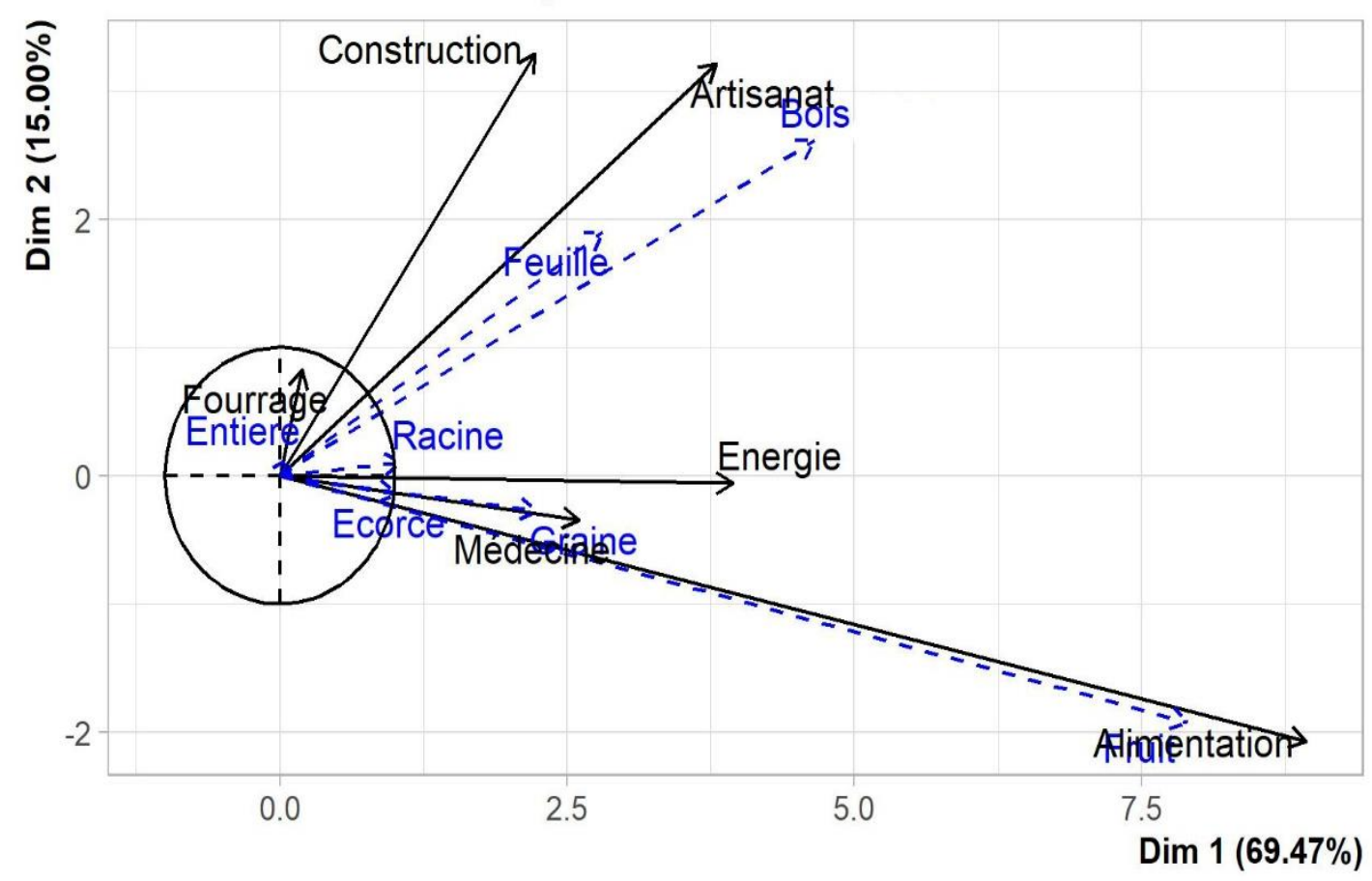

Figure 3 : Graphe de l'ACP sur les utilisations et les parties utilisées des plantes. 


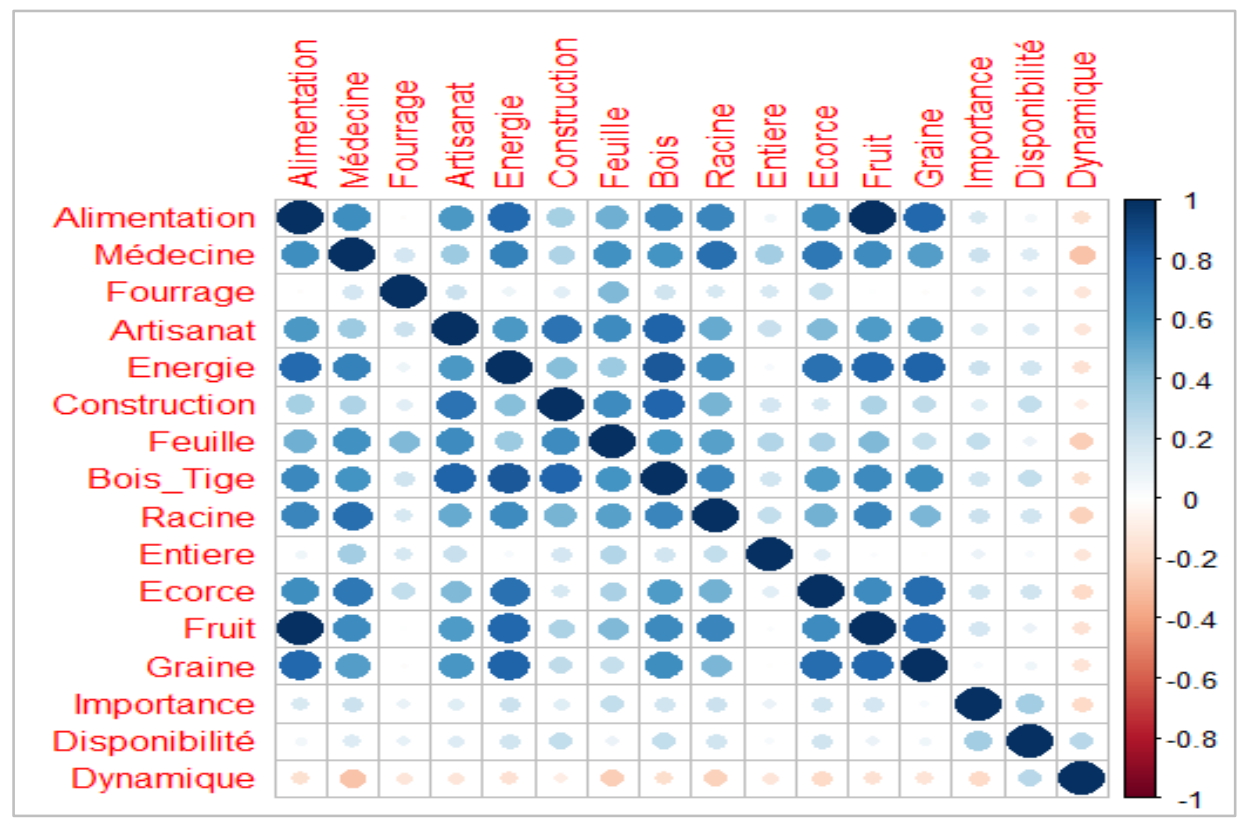

Figure 4 : Matrice de corrélation entre les utilisations et les parties utilisées des espèces végétales.

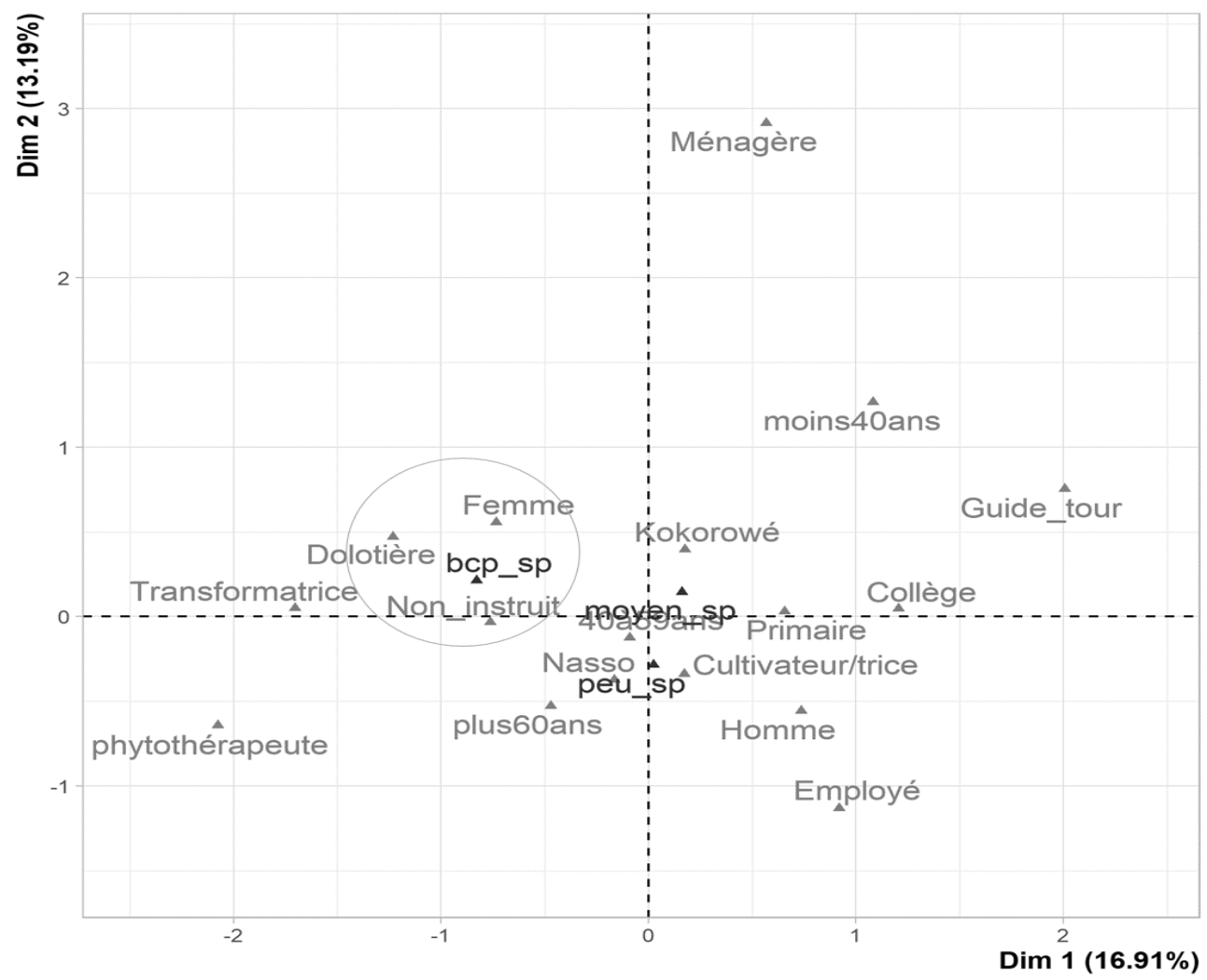

Bcp_sp :utilisation de beaucoup d'espèces (plus de 20 espèces), peu_sp : utilisation de peu d'espèces (moins 10 espèces) ; moyen_sp : utilisation moyenne des espèces (entre 10 et 19 espèces). Guide_tour : guide touristique.

Figure 5 : Graphe de l'ACM sur l'utilisation des espèces végétales en fonction des facteurs socioprofessionnels. 


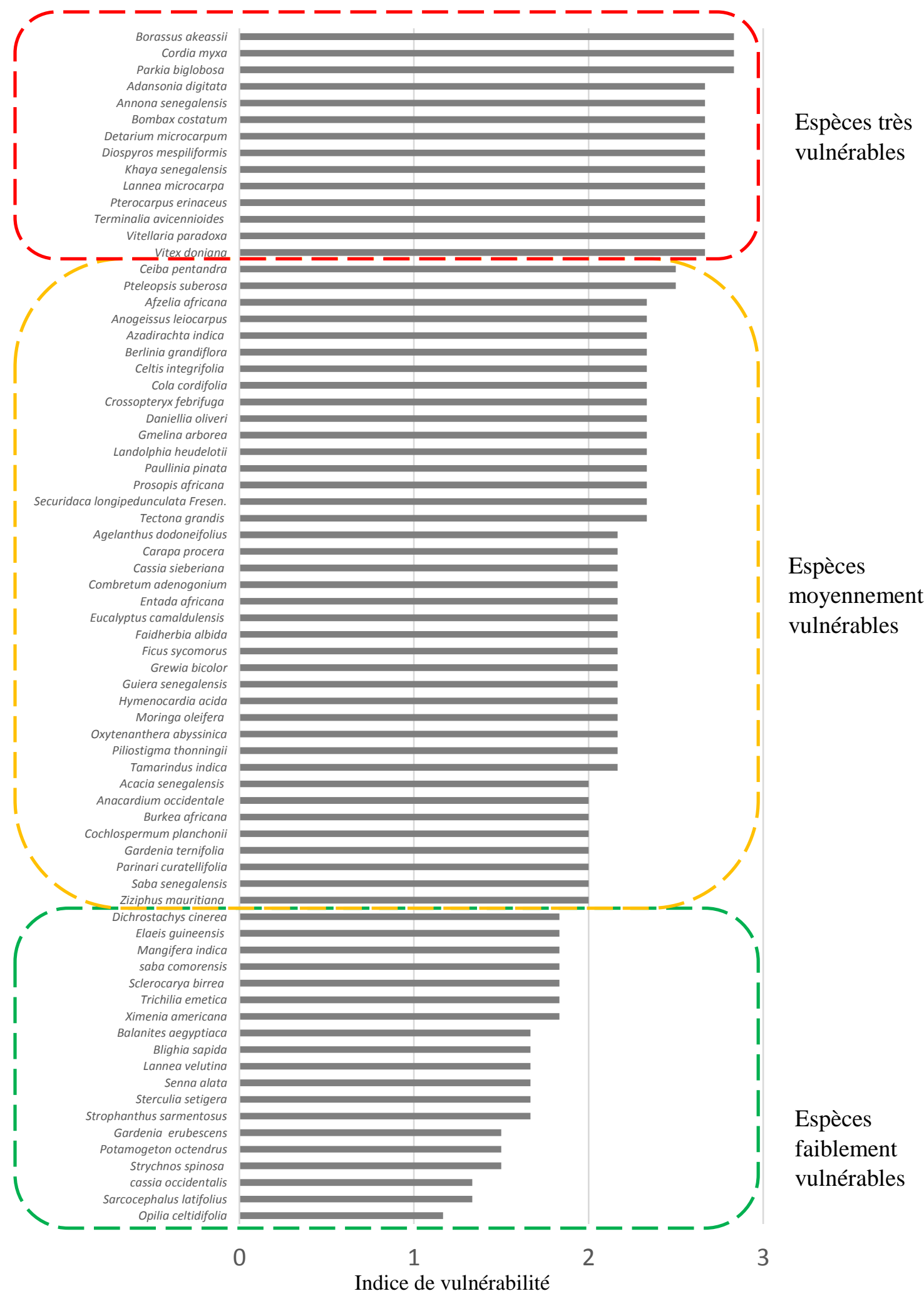

Figure 6 : Spectre de vulnérabilité des espèces utilisées par les populations riveraines de FCK. 


\section{DISCUSSION}

\section{Diversité des espèces utilisées et leur disponibilité dans la FCK}

Les ressources forestières constituent une source importante d'approvisionnement pour les populations riveraines des aires protégées. L'étude a permis de recenser 80 espèces utilisées par la population riveraine de la FCK. 74,04\% de ces espèces se retrouvent dans la FCK qui compte 206 espèces. Ce qui montre que les formations végétales protégées offrent une grande potentialité et contribuent énormément à la satisfaction des besoins essentiels des populations rurales. Comparé aux études antérieures dans la région, le nombre d'espèces (80) utilisées est supérieur aux 73 espèces recensées par Traoré et al. (2011) au Sud-Ouest du Burkina Faso. Cette différence peut s'expliquer par le fait que la présente étude a pris en compte aussi bien les ligneux que les herbacées. Au niveau de la composition floristique on note une similarité entre la flore citée et celle inventoriée car elles sont toutes deux dominées par la famille des Fabaceae et ont en commun $74,04 \%$ des espèces utilisées. Selon Traoré et al. (2011), ceci montre que la population riveraine de la FCK a une bonne connaissance de son environnement et traduit également la fiabilité des enquêtes ethnobotaniques comme moyen pour une estimation rapide de l'état de la végétation d'une localité.

\section{Utilisation des espèces végétales par la population riveraine de la FCK}

Dans les villages de Nasso et de Kokorowé les plantes sont plus utilisées pour l'alimentation et la médecine. Cela pourrait s'expliquer par le fait que les populations, dans leur grande majorité, sont tributaires de la médecine traditionnelle locale, à cause de leur niveau de revenu moyen et des habitudes socioculturelles. Selon Lawin et al. (2016) l'usage traditionnel des plantes médicinales constitue la base de la médecine curative des populations à revenu modeste. Selon Yelkouni et CharassePouele (2006), la culture des soins basée sur l'usage des ressources naturelles constitue un élément important de santé publique dans beaucoup de pays africains pour des raisons historique, culturelle et sociale. De plus le coût généré par l'utilisation des plantes est relativement moins chère que le coût des prestations de soins et des médicaments dans les centres de santé. L'utilisation des fruits par tous montre leur importance dans l'alimentation de la population et peut s'expliquer par leur disponibilité dans les parcs agroforestiers mais aussi, par le fait que leur vente procure des revenus substantiels pour un bon nombre de ménages. Les fruits de Vitellaria paradoxa, Parkia biglobosa et Adansonia digitata font de plus en plus l'objet de transactions commerciales importantes à l'échelle nationale et internationale. $\mathrm{Au}$ Burkina Faso, Vitellaria paradoxa (le karité) est le troisième produit d'exportation après le coton et les produits de l'élevage (Badini et al., 2011). Les fruits de Detarium microcarpum, Lannea microcarpa, Saba senegalensis et Adansonia digitata sont vendus sur les marchés des villes environnantes pour une consommation directe mais aussi pour la production de jus de fruits naturels très appréciés par les citadins. Ainsi, au niveau local, ces PFNLs constituent une importante source de revenus aux différentes composantes de la communauté, notamment les femmes, qui sont les plus impliquées dans la cueillette ou le ramassage et la vente de ces fruits. Outre les PFNLs, le bois fait partie des produits les plus sollicités $(93,55 \%)$. Cela peut s'expliquer par sa forte demande dans plusieurs commodités d'usage que sont l'artisanat, la construction des maisons et surtout le bois d'énergie. En effet, le combustible ligneux constitue la principale source d'énergie pour la cuisson à Nasso et à Kokorowé surtout pour la préparation de la bière locale appelée « dolo » qui nécessite une très grande quantité de bois. Ceci expliquerait les résultats de l'ACM et du V-test de Cramer qui mettent en évidence un fort lien entre l'utilisation de beaucoup d'espèces et cette profession. 


\section{Influence des facteurs socio-professionnels sur l'utilisation des espèces végétales}

L'utilisation des plantes à Nasso et à Kokorowé semble être influencée fortement par la profession, le sexe et le niveau académique des populations. En effet, les résultats de l'ACM montrent que les femmes, les personnes non instruites et les productrices de bière locale utilisent beaucoup d'espèces. Le facteur fondamental qui justifie cette forte utilisation des plantes dans notre étude est l'activité de production de bière locale (Vtest $>1$, p-value $<0,05)$ donc la profession. En effet, cette activité est conduite uniquement par les femmes qui sont pour la plupart non instruites. Ce résultat confirme certaines études antérieures qui ont montré que les connaissances ethnobotaniques sont globalement influencées par le sexe (Lougbegnon et al., 2011), le niveau d'étude (Dassou et al., 2015) et la profession des enquêtés (Beltràn-Rodriguez et al., 2014). Ceci rappelle l'importance des femmes dans la gestion des ressources forestières. Tandis qu'elles jouent un rôle clé dans l'exploitation des ressources forestières, elles sont généralement ignorées lors de la mise en place des politiques forestières. Cela entraine une faible prise en compte de leurs besoins et intérêts stratégiques lors de la mise en œuvre des plans de gestion (Bauer, 2010). Ceci n'est cependant pas sans conséquence sur la vulnérabilité des plantes qu'elles utilisent.

\section{Vulnérabilité des espèces végétales utilisées}

Le spectre de vulnérabilité réalisé à partir des données recueillies montre que la pression sur les ressources du fait de leur importance socio-économique les rend plus vulnérables. Ces résultats sont similaires à ceux de Traoré et al. (2011) dans la même zone phytogéographique, qui ont trouvé 60 espèces vulnérables et 15 espèces très vulnérables comparées respectivement aux 55 et 14 espèces dans la présente étude. Sur les 14 espèces très vulnérables identifiées, 11 sont déjà classées parmi les espèces menacées du Burkina Faso (Thiombiano et al., 2010) et 11 autres sur la liste rouge de l'UICN (2020). Ainsi, l'indice de vulnérabilité peut être un outil efficace et rapide pour évaluer l'état de la phytodiversité d'une localité, d'une région ou d'un pays. De façon globale, le mode de collecte et les organes végétaux utilisés, semblent être les principales causes de cette vulnérabilité. Ainsi, la récolte intensive des fruits (Vitellaria paradoxa, Borassus akeassii) ou des graines (Parkia biglobosa), des racines (Detarium microcarpum), des fleurs (Bombax costatum) et des écorces (Parkia biglobosa, Trichilia emetica), entraînerait une diminution progressive des espèces qui les produisent. Du point de vue de la population, toutes les catégories d'usage et modes d'exploitation et plus particulièrement l'utilisation médicinale et l'exploitation des feuilles, des racines et des écorces, ont un impact négatif sur la dynamique des espèces utilisées. Selon Guigma et al. (2012), chaque partie exploitée a un inconvénient sur la survie de l'espèce. De ce fait, toutes les espèces à utilisations diverses et aux indices élevés d'utilisation mériteraient des attentions particulières dans les stratégies de gestion des espèces spontanées.

\section{Perceptions et stratégies d'adaptation des populations de la FCK}

Le fait que la majeure partie de la population enquêtée soit consciente des risques de perte de la diversité floristique $(71,43 \%)$ est dû à sa dépendance vis-à-vis des ressources forestières. Selon le Secrétariat de la Convention sur la diversité biologique (2009), les pauvres sont particulièrement vulnérables aux conséquences de la perte de la biodiversité car ils sont souvent directement dépendants de la diversité biologique pour leur survie quotidienne et n'ont pas les moyens de se procurer des produits de substitution.

Quant à la substitution des espèces comme stratégie d'adaptation, plusieurs études ont montré, que les populations optent, soit, pour un remplacement d'une plante par une autre, soit, pour l'utilisation d'une partie à la place d'une autre partie de la même plante (Kaboré et al., 2015). Le nombre d'espèces de 
substitution proposées (11) est largement inférieur au nombre d'espèces vulnérables identifiées (55). De plus, l'utilisation de Vitellaria paradoxa et Parkia biglobosa comme substitut n'est pas acceptable, car, elles sont déjà très vulnérables dans la localité (IVi $\geq 2,5$ ) et figurent parmi les espèces menacées. Parmi les substituts, il y a 5 espèces ligneuses exotiques: Anacardium occidentale, Tectona grandis, Eucalyptus camaldulensis, Azadirachta indica, et Gmelina arborea. Leur sylviculture pourrait être encouragée afin de réduire la pression sur les formations naturelles. Toutefois, l'utilisation de Eucalyptus camaldulensis doit être encadrée car certaines études ont montré que la monoculture des Eucalyptus présente des risques écologiques et agronomiques tels que l'appauvrissement des sols, l'épuisement des nappes phréatiques et la diminution de la biodiversité en occurrence les espèces du sousbois (Soumare et al., 2017; Bayle, 2019). Selon Soumare et al. (2017), les pieds de Eucalyptus présentent également des effets allélopathiques négatifs qui inhibent la germination et la croissance de certaines espèces végétales avec également des effets microbicides ou inhibiteurs sur des communautés microbiennes du sol. C'est pour cela il serait mieux de privilégier la sylviculture des espèces locales prisées ou leur conservation par l'agroforesterie. En effet, certaines espèces locales continueront à être exploitées quelle que soit leur vulnérabilité à cause des habitudes culturelles. Par exemple, les graines de Glycine max (Soja) proposées pour remplacer les graines de Parkia biglobosa dans la fabrication du « soumbala », une épice locale très prisée, n'a pas reçu une adhésion favorable de la population à cause de la différence organoleptique et surtout de l'habitude alimentaire. C'est pour cela, 58,82\% des enquêtés ont adopté comme stratégie d'adaptation l'agroforesterie. Selon Bationo (2010), les paysans ont toujours conservé ou voulu conserver dans leurs champs certaines espèces ligneuses qu'ils jugent nécessaires à leur bien-être. Ce maintien volontaire des ligneux dans les champs favorise leur protection, assure une production durable des écosystèmes agricoles et une diversification de la production.

\section{Conclusion}

Cette étude a permis d'appréhender l'importance socioéconomique des ressources végétales pour la population riveraine de la FCK. Au total 80 espèces sont utilisées par la population sur 206 espèces présentes dans la FCK. L'étude montre que les forêts protégées offrent une grande potentialité pour les populations riveraines et contribuent à la satisfaction de leurs besoins essentiels tels que l'alimentation et la médecine. Les PFNLs sont aussi une importante source de revenus pour la population, en l'occurrence les femmes. Vue leur rôle important dans l'exploitation de ces ressources, les femmes et les personnes non instruites doivent être prises en compte dans l'élaboration et la mise en œuvre des politiques de gestions forestières.

L'évaluation de la vulnérabilité des ressources forestières a permis de dresser une liste d'espèces ligneuses vulnérables de la région et d'identifier les principales causes de leur vulnérabilité à savoir le mode de collecte et les organes végétaux utilisés. Bien que cette liste soit relative et non exhaustive, l'approche peut aider à identifier rapidement les menaces qui pèsent sur les espèces dans une localité afin de mieux sensibiliser les populations. À cet effet, la population riveraine de la FCK est bien consciente de la vulnérabilité des espèces utilisées et propose donc l'agroforesterie pour leur conservation. Toutes fois, en plus de cette stratégie, on peut leur recommander la sylviculture à petite échelle des espèces ligneuses locales proposées par certains comme substitut de ces espèces vulnérables.

\section{CONFLIT D'INTERETS}

Les auteurs déclarent qu'il n'y a aucun conflit d'intérêts.

\section{CONTRIBUTIONS DES AUTEURS}

YCR a élaboré le protocole, effectué la collecte et l'analyse des données. SYJ a participé à la collecte des données. HM, KSA 
et SI ont supervisé la rédaction du protocole et du manuscrit.

\section{REMERCIEMENTS}

Nous remercions «IFS - International Foundation for Science » qui a financé cette étude, à travers sa bourse de recherche individuelle ( $\mathrm{N}^{\circ} \mathrm{IFS}$ : I-1-D-6350-1). Nos remerciements vont également à l'endroit des populations des villages de Nasso et de Kokoroué particulièrement et notre guideinterprète Mr. Sanou Bakary pour leur accueil et disponibilité lors des travaux de terrain.

\section{REFERENCES}

Arbonnier M. 2009. Arbres, Arbustes et Lianes des Zones Sèches d'Afrique de l'Ouest ( $3^{\text {ème }}$ edn). Quae, Muséum National d'Histoire Naturelle(MNHN): Paris, France.

Badini Z, Kaboré M, Mheen-Sluijer JVD, Vellema S. 2011. Historique de la filière karité au Burkina Faso et des services offerts par les partenaires techniques et financiers aux acteurs. Value Chains for Pro-poor Development Program Research Paper. Wageningen University and Research Centre. p.38.

Bationo BA. 2010. Utilisation des terres: Systèmes et pratiques agroforestiers. In Atlas de la Biodiversité de l'Afrique de l'Ouest Tome II, Burkina Faso. Thiombiano A, Kampmann D (eds). Ouagadougou \& Frankfurt/Main ; 252255.

Bauer L. 2010. Forêts et réduction de la pauvreté dans les pays en développement : une relation à déchiffrer. Essai de master en en ingénierie et management de l'environnement et du développement durable, Université de Sherbrooke, Sherbrooke, p.119.

Bayle GK. 2019. Ecological and social impacts of Eucalyptus tree plantation on the environment. J. Biodivers. Conserv. Bioresour. Manag. 5(1): 93-104. DOI: https://doi.org/10.3329/jbcbm.v5i1 .42189
Beltrán-Rodríguez L, Ortiz-Sánchez A, Mariano NA, Maldonado-Almanza B, Reyes-García V. 2014. Factors affecting ethnobotanical knowledge in a mestizo community of the Sierra de Huautla Biosphere Reserve, Mexico. Journal of Ethnobiology and Ethnomedicine, 10 : 218. DOI : https://doi.org/10.1186/17464269-10-14

Berhaut J. 1967. Flore du Sénégal (2 ${ }^{\mathrm{ème}}$ edn). Clairafrique : Dakar, Sénégal.

Betti JL. 2001. Vulnérabilité des plantes utilisées comme antipaludiques dans l'arrondissement de Mintom au Sud de la réserve de Biosphère du Dja (Cameroun). Syst. Geogr. Pl., 71: 661- 678. DOI: https://www.jstor.org/stable/3668709.

CIFOR (Center for International Forestry Research). 2005. Les forêts et le développement de l'Afrique. CIFOR. DOI :

https://doi.org/10.17528/cifor/001779.

Cotton CM,1996. Ethnobotany: Principles and Applications. John Wiley \& Sons Ltd : Chichester, Royaume-Uni .

Daget P, Poissonet J. 1990. Notion de valeur pastorale. Repères, $2:$ 4-8.

Dassou HG, Yedomonhan H, Adomou AC, Ogni CA, Tossou MG, Akoegninou A. 2015. Facteurs socioculturels et environnementaux déterminant la connaissance ethno vétérinaire au Bénin. Afrique Science, 11(5) : 335-360. DOI:

DIFOR (Direction des Forêts), 2007. Situation des forêts classées du Burkina Faso et plan de réhabilitation. DIFOR. Burkina Faso, p. 46.

Guigma Y, Zerbo P, Millogo-Rasolodimby J. 2012. Utilisation des espèces spontanées dans trois villages contigus du Sud du Burkina Faso. Tropicultura, 30(4) : 230235.

DOI : http://www.tropicultura.org/text/v30n4/2 30.pdf

Guinko S. 2005. Flore illustrée de la forêt classée du Kou. Projet d'aménagement participatif des forêts classées de 
Dindéresso et du Kou. Burkina Faso, p. 134.

Hadonou-Yovo AG, Houessou LG, Lougbegnon TO, Adebi Y, Sinasson GKS, Semevo DF, Udo Lange U, Boko M. 2019. Diversité et formes d'utilisation des espèces ligneuses de la Réserve de biosphère du Mono (Bénin). Vertigo, la revue électronique en science de l'environnement, 19(2). DOI : https://doi.org/10.4000/vertigo.26257 .

Hahn-Hadjali K, Thiombiano A. 2000. Perception des espèces en voie de disparition en milieu Gourmantché (Est du Burkina Faso). Berichte des Sonderforschungsbereichs, 268 : 285297.

Houéhanou DT, Assogbadjo AE, Chadare FJ, Zanvo S, Sinsin B. 2016. Approches méthodologiques synthétisées des études D'ethnobotanique quantitative en milieu tropical. Spécial Projet Undesert-UE. Annales des Sciences Agronomiques, 20 : 187-205.

INSD (Institut National de la Statistique et de la Démographie). 2020. Cinquième Recensement Général de la Population et de l'Habitation du Burkina Faso. Résultats Préliminaires. INSD : Ouagadougou, Burkina Faso, p. 76.

Kaboré SA, Schumann K, Hien M, Lykke AM, Hahn K, Nacro HB. 2015. Stratégies d'adaptation à la réduction des services écosystémiques: cas des potentialités de substitution de trois espèces forestières dans le Sud-ouest du Burkina Faso. Int. J. Biol. Chem. Sci., 9(3): 1194 -1208. DOI: 10.4314/ijbcs.v9i3.5

Lawin IF, Lalèyè OAF, Agbani OP. 2016. Vulnérabilité et stratégies endogènes de conservation des plantes utilisées dans le traitement du diabète dans les communes de Glazoué et Savè au Centre-Bénin. Int. J. Biol. Chem. Sci., 10(3): 1069-1085. DOI: 10.4314/ijbcs.v10i3.14

Lebrun JP, Stork A. 1991. Enumération des plantes à fleurs d'Afrique tropicale. I Généralités et Annonaceae à Pandaceae,
Conservatoire et Jardin botaniques de la ville, Genève, $250 \mathrm{p}$.

Loubelo E. 2012. Impact des produits forestiers non ligneux (PFNL) sur l'économie des ménages et la sécurité alimentaire : cas de la République du Congo. Thèse de doctorat en économie. Université Rennes 2. France, p. 260.

Lougbegnon TO, Tente BAH, Amontcha M, Codjia JTC. 2011. Importance culturelle et valeur d'usage des ressources végétales de la réserve forestière marécageuse de la vallée de Sitatunga et zones connexes. Bulletin de la Recherche Agronomique du Bénin, 70 : 35-46.

Lykke AM, Kristensen MK, Ganaba S. 2004. Valuation of local use and dynamics of 56 woody species in the Sahel. Biodiversity and Conservation, 13: 1961-1990. DOI : https://doi.org/10.1023/B:BIOC.0000035 876.39587.

Ouédraogo-Belem M. 2008. Les galeries forestières de la Réserve de la Biosphère de la Mare aux Hippopotames du Burkina Faso : caractéristiques, dynamique et ethnobotanique. Thèse de Doctorat d'Etat ès Sciences Naturelles, Université de Ouagadougou. Burkina Faso, p. 262.

Ouédraogo O, Ouédraogo A, Thiombiano A. 2010. La forêt classée du Kou: une aire protégée d'importance capitale. In Atlas de la Biodiversité de l'Afrique de l'Ouest Tome II, Burkina Faso, Thiombiano A, Kampmann D (eds). Ouagadougou \& Frankfurt/Main ; 373-385.

Ozer P, Gassani J, Hountondji YC, Niang AJ, Ambouta K. 2007. La désertification estelle en recul au Sahel ? Bois et Forêts des Tropiques, 293(3) : 23-28.

Savadogo S, Ouoba PA, Da DEC. 2019. Extraction des PFNL et menace sur les espèces pourvoyeuses au Burkina Faso : Cas de Kokologho et Tenado dans la région du centre-ouest. Rev. Ivoir. Sci. Technol., 33 : 240-258.

Secrétariat de la Convention sur la diversité biologique (CDB). 2009. Biodiversité, développement et réduction de la 
pauvreté : reconnaitre le rôle de la biodiversité pour le bien-être humain. Montréal. p.52.

Soumare A, Diedhiou AG, Kane A. 2017. Les plantations d'Eucalyptus au Sahel : distribution, importance socioéconomique et inquiétude écologique. Int. J. Biol. Chem. Sci., 11(6): 3005-3017.

DOI: https://dx.doi.org/10.4314/ijbcs.v11i6.36 Tankoano B, Hien M, Dibi NH, Sanon Z, Yameogo TJ, Somda I, 2015. Dynamique spatio-temporelle des savanes boisées de la forêt classée de Tiogo au Burkina Faso. Int. J. Biol. Chem. Sci., 9(4) : 1983-2000. DOI: $10.4314 /$ ijbcs.v9i4.23

Thiombiano A, Glèlè Kakaï R, Bayen $\mathrm{P}$, Boussim JI, Mahamane A. 2016. Méthodes et dispositifs d'inventaires forestiers en Afrique de l'Ouest : état des lieux et propositions pour une harmonisation. Annales des Sciences Agronomiques, 20 : 15-31.

Thiombiano A, Schmidt M, Da S, HahnHadjali K, Zizka G, Wittig R. 2010. Les plantes vasculaires : Les plantes à fleurs. In Atlas de la Biodiversité de l'Afrique de l'Ouest Tome II, Burkina Faso, Thiombiano A, Kampmann D (eds). Ouagadougou \& Frankfurt/Main; 184192.

Traoré L, Ouedraogo I, Ouedraogo A, Thiombiano A. 2011. Perceptions, usages et vulnérabilité des ressources végétales ligneuses dans le Sud-Ouest du Burkina Faso. Int. J. Biol. Chem. Sci., 5(1): 258278. DOI : $10.4314 /$ ijbcs.v5i1.68103.

UICN (Union Internationale pour la Conservation de la Nature). 2020. Liste rouge des espèces menacées. https://www.iucnredlist.org/fr/. 